\title{
An episomal CRISPR/Cas9 system to derive vector-free gene modified mammalian cells
}

\section{Dear Editor,}

CRISPR and CRISPR-associated (Cas) proteins play their adaptive immunity role in degrading foreign nucleic acids in both bacteria and archaea. CRISPR/Cas has proved efficient in modifying mammalian genomes (Cong et al., 2013; Mali et al., 2013) and various delivery methods for Cas9: gRNA complex have been established. Among different delivery means, in vitro transcribed Cas9 mRNA/gRNA, purified Cas9 protein, and adeno-associated virus (AAV) driven seem promising toward clinical applications (Kouranova et al., 2016; Lin et al., 2014; Yin et al., 2016). Still, all the fore-mentioned methods for delivering CRISPR/Cas9 have certain shortcomings. In vitro transcription and protein purification are complicated and inconvenient for use, while AAV mediated system suffers from the side effects of immune response and insertional mutagenesis (Donsante et al., 2007; Li et al., 2011).

Episomal vectors based on oriP-EBNA1 have been shown as an effective method to derive vector-free cells in reprogramming studies (Okita et al., 2011; Yu et al., 2009). Without selection pressure, EBV vectors are gradually lost in each generation due to their defects in synthesizing and partitioning thus resulting in transgene-free daughter cells (Nanbo et al., 2007). We thus set out to test if Cas9 protein and gRNA can be effectively delivered as episomes to generate vector-free mutations in mammalian cells. Here we report efficient human genome editing with CRISPR/Cas9 technology in the form of oriP-EBNA1-based episomes. To obtain oriP-EBNA1-based CRISPR/Cas9 vectors, we first assembled CMV-FLAG-hSpCas9-2A-GFP and U6-gRNA and oriP-EBNA1 fragments together. To further enrich successfully transfected cells, we added SV40-puro to form pCRISPR-S12 (Fig. 1), which hereafter is referred to as oriPEBNA1-based CRISPR/Cas9 vector or system.

To test the oriP-EBNA1-based CRISPR/Cas9 system, we first examined its efficiency for disrupting single-copy tdTomato expression driven by CAG promoter in mouse induced pluripotent stem cells (miPSCs). We designed crtdTomato to target tdTomato coding sequence with the expectation of destructing an existing Sau3Al restriction enzyme site (Fig. 2A). The tdTomato-tagged-miPSCs were transfected with PCRISPR-S12-crtdTomato and manipulated according to the flowchart (Fig. 2B). We observed disappearance of tdToamto fluorescence in some of the miPSCs clones under a fluorescent microscope (Fig. 2C). Further, flow-activated cell sorting (FACS) showed a reduction of $26 \%$ tdTomatolabeled cells in crtdTomato treated group when compared with non-transfected group five days post transfection (Fig. 2D). These results suggested that our system works in inactivating reporter genes in miPSCs.

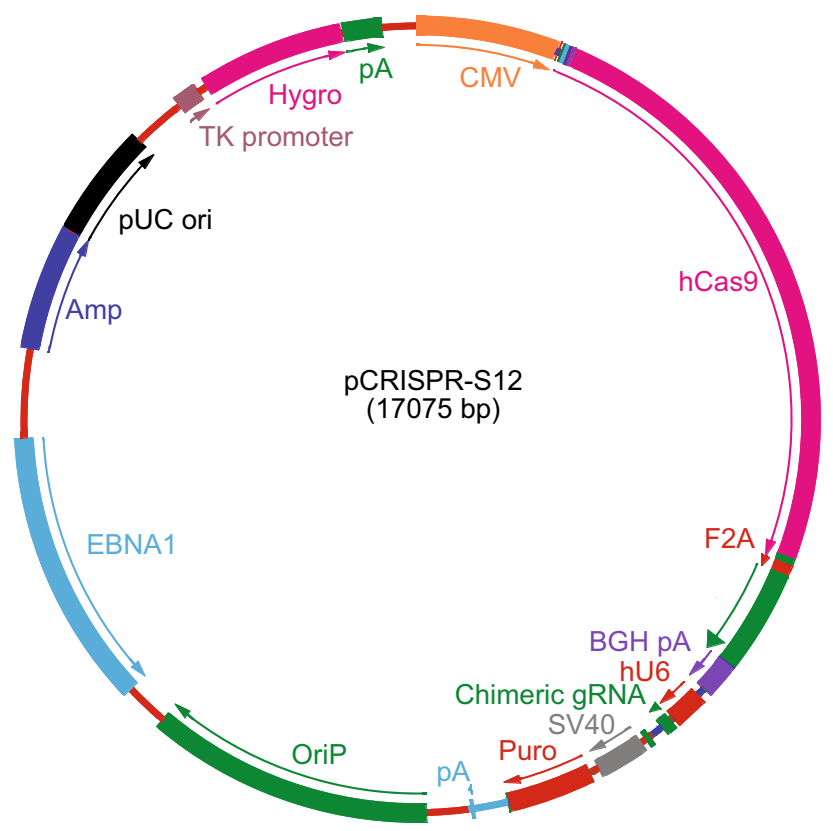

Figure 1. Circular map of oriP-EBNA1-based CRISPR/ Cas9 vector. CMV, cytomegalovirus promoter; $3 \times$ FLAG, FLAG epitope tag; hCas9, humanized Streptococcus pyogenes Cas9; F2A, 2A regions of foot-and-mouth disease virus; GFP, green fluorescent protein gene; $B G H$ pA, bovine growth hormone polyA signal; U6, human U6 promoter; chimeric gRNA, synthetic gRNA scaffold; oriP, Epstein-Bar virus (EBV) latent origin of replication; EBNA1, EBV nuclear antigen-1, replication transactivator of EBV; SV40, SV40 promoter; Puro, puromycin resistant gene; Amp, ampicillin resistant gene; pUC ori, replication origin; TK promoter, thymidylate kinase promoter; Hygro, hygromycin resistant gene; $\mathrm{pA}$, polyA signal. 
A

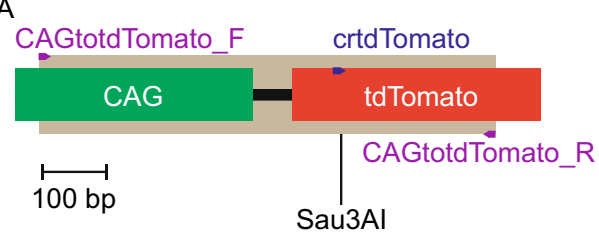

C
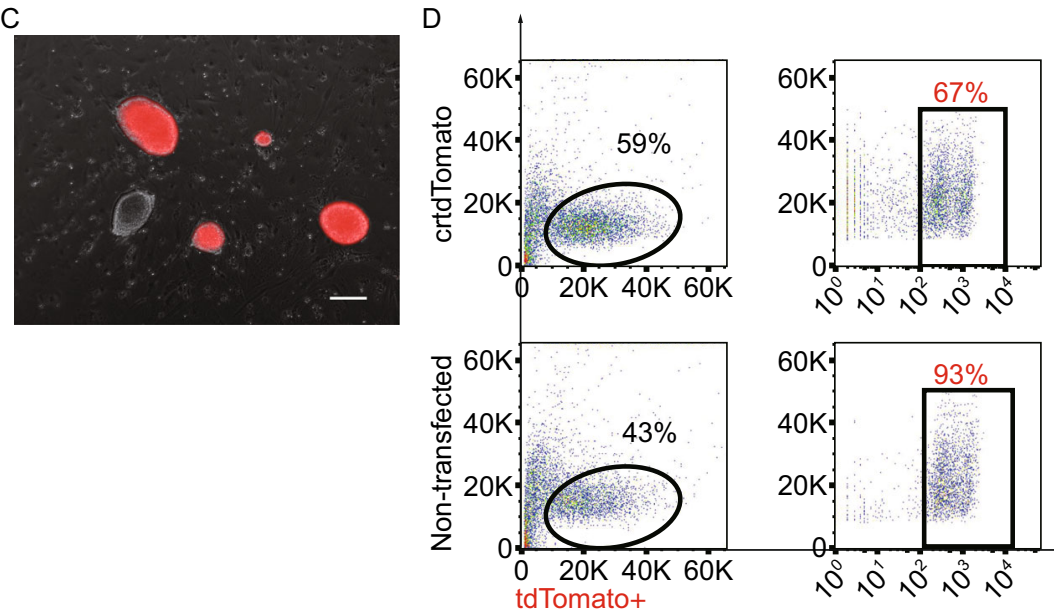

$E$

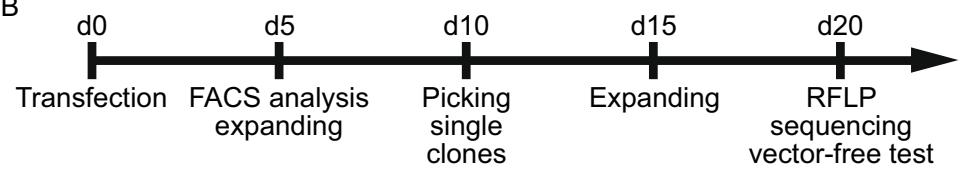

$\mathrm{F}$
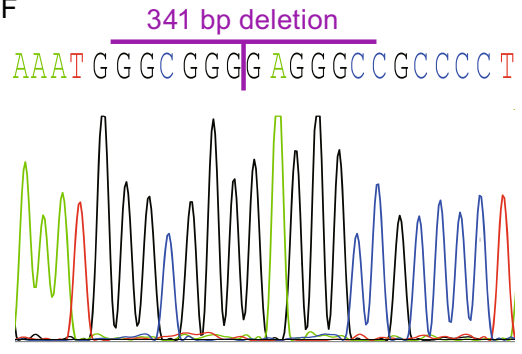

G

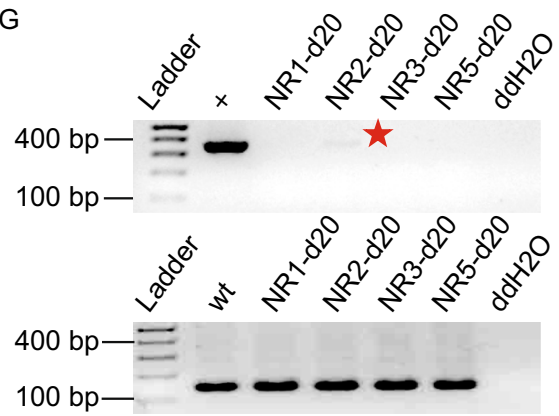

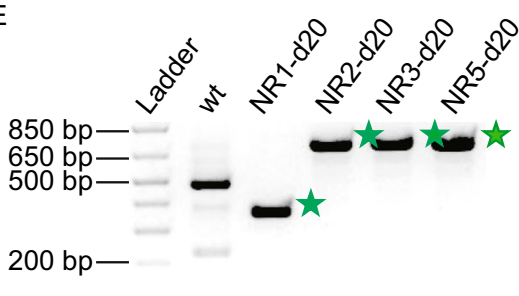

$\mathrm{H}$

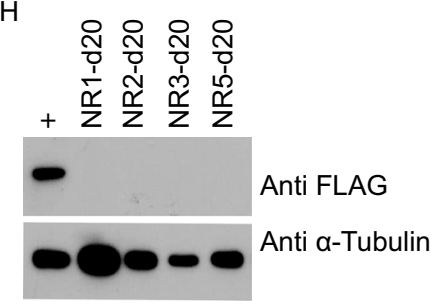

Figure 2. oriP-EBNA1-based CRISPR/Cas9 disrupted fluorescent expression in miPSCs. (A) Schematic of crtdTomato targeting site (blue arrow), primers used (purple arrows) for amplifying genomic sequences flanking target sites (light gray boxes), CAG promoter (green box) drove the expression of tdTomato (red box), and Sau3Al restriction sites were used for RFLP assay. (B) Schematic of the experimental procedure. (C) Disappearance of red fluorescence in partial tdTomato-labelled-miPSCs clones. Scale bar, $200 \mu \mathrm{m}$. (D) FACS analysis of crtdTomato transfected and non-transfected miPSCs. The upper two panels are crtdTomato transfected group (tdTomato + with a portion of 67\%) and the lower two panels are non-transfected group (tdTomato+ with a portion of $93 \%$ ). (E) RFLP of non-red (NR) clones by Sau3Al. The green pentagrams indicated the uncut PCR amplicons by Sau3AI. Ladder, $1 \mathrm{~kb}$ plus ladder in all figures in this study. $(\mathrm{F})$ Sanger sequencing confirmed deletion of $341 \mathrm{bp}$ of NR1-d20 clone. The purple line between G/G showed the deleted site. (G) PCR confirmed the removal of EBNA1 fragment in clone NR1-d20, NR2-d20, NR5-d20 but not in NR3-d20. pCRISPR-S12 DNA was used as positive control (“+" in all figures in this study). "wt" stands for wild type mouse genomic DNA. Red pentagram shows visible EBNA1 residue in NR3-d20. (H) Western blot showed no hSpCas9 expression in clone NR1-d20, NR2- d20, NR3-d20, and NR5- d20. HeLa cells expressing FLAG-hSpCas9-2A-GFP stably was used as positive control ("+" in all figures in this study). The expected protein band is about $190 \mathrm{kDa}$.

Next, we further detected the targeting efficiency of our system in 6 non-red (NR) single clones. We were able to PCR amplify the target regions in 4 out of the 6 NR clones. Clones NR4 and NR6 were not analyzed further since PCR failed to produce products (Fig. S1). PCR products of the remaining 4 clones were confirmed as expected mutants by restriction fragment length polymorphism (RFLP) assays (Fig. 2E and 2F). Among these, clone NR1 showed a truncated size (Fig. S1), which was further confirmed by Sanger sequencing as a 341 nucleotides deletion (Fig. 2F). Above data showed that our oriP-EBNA1-based CRISPR/Cas9 system could mediate efficient gene disruption. 
Finally, we examined whether these four successfully engineered miPSCs (NR1, NR2, NR3, NR5) were free of targeting vector. PCR analysis showed that 3 clones were free of vectors, but clone NR2-d20 still retained residual amount of vectors (Fig. 2G). However, Western blot of FLAG-hSpCas9-2A-GFP further verified no continuous expression of hSpCas9 protein of all four clones (Fig. $2 \mathrm{H}$ ), suggesting that PCR detection was more sensitive in detecting foreign gene expression than Western blot assay. Together, these results demonstrated that our oriP-EBNA1based CRISPR/Cas9 system inactivated reporter gene expression as expected, and more importantly, vector-free engineered cell lines can be readily obtained.

We further attempted to examine whether our system works well in human cells. The basic functions of CRISPR/ Cas 9 were confirmed by deleting accurately more than $2 \mathrm{~kb}$ intervening nucleotides of human chemokine receptor 5 (CCR5) as well as editing multiplex genes (human TET1, $T E T 2$, and TET3) simultaneously (Figs. S3-S6 and Table S1). Moreover, our oriP-EBNA1-based CRISPR/Cas9 system was free of vector in both genetically modified miPSCs and human cells. Together, these results demonstrated the feasibility of manipulating mouse and human genomes with our oriP-EBNA1-based CRISPR/Cas9 system.

In the current study, we have verified the functionality of our episomal CRIPSR/Cas9 system first by inactivating visible reporter gene, and further by specifically deleting a single genomic region and editing multiplex genes in a transgene-free manner.

One potential risk for CRISPR/Cas9 in genome editing is its uncertain off-target effects which could confound genome-editing-based therapies. In present study, we detected certain frequencies (none for crTETs and 10\% for crCCR5) of off-target events by T7El assay (Fig. S7 and Table S2). In the future, combined with other progresses, such as mutant Cas9 or new homologues and optimized gRNA design, episomal CRISPR/Cas9 could further ameliorate, if not eliminate, the off-target influences, thereby broadening its application.

\section{FOOTNOTES}

Linlin Li conceived and designed the experiments. Linlin Li and Fei Gao performed the experiments. Linlin Li, Fei Gao, and Sen Wu wrote the paper. We are grateful for continuous support from the $\mathrm{Wu}$ lab members. This work was supported by the National Natural Science Foundation of China (Grant No. 31271598) and The Project for Extramural Scientists of State Key Laboratory of Agrobiotechnology (2015SKLAB6-15). Linlin Li, Fei Gao, and Sen Wu declare no

Linlin Li and Fei Gao contributed equally to this article.

Electronic supplementary material The online version of this article (doi:10.1007/s13238-016-0299-9) contains supplementary material, which is available to authorized users. conflict of interest. This article does not contain any studies with human or animal subjects performed by the any of the authors.

Linlin $\mathrm{Li}^{1}$, Fei Gao ${ }^{1}$, Sen $\mathrm{Wu}^{1 凶}$

${ }^{1}$ State Key Laboratory of Agrobiotechnology, College of Biological Sciences, China Agricultural University, Beijing 100193, China

$\bowtie$ Correspondence: swu@cau.edu.cn (S. Wu)

\section{OPEN ACCESS}

This article is distributed under the terms of the Creative Commons Attribution 4.0 International License (http://creativecommons.org/ licenses/by/4.0/), which permits unrestricted use, distribution, and reproduction in any medium, provided you give appropriate credit to the original author(s) and the source, provide a link to the Creative Commons license, and indicate if changes were made.

\section{REFERENCES}

Cong L, Ran FA, Cox D, Lin S, Barretto R, Habib N, Hsu PD, Wu X, Jiang W, Marraffini LA et al (2013) Multiplex genome engineering using CRISPR/Cas systems. Science 339:819-823

Donsante A, Miller DG, Li Y, Vogler C, Brunt EM, Russell DW, Sands MS (2007) AAV vector integration sites in mouse hepatocellular carcinoma. Science 317:477

Kouranova E, Forbes K, Zhao G, Warren J, Bartels A, Wu Y, Cui X (2016) CRISPRs for optimal targeting: delivery of CRISPR components as DNA, RNA and protein into cultured cells and single-cell embryos. Hum Gene Ther 27:464-475

Li H, Malani N, Hamilton SR, Schlachterman A, Bussadori G, Edmonson SE, Shah R, Arruda VR, Mingozzi F, Wright JF et al (2011) Assessing the potential for AAV vector genotoxicity in a murine model. Blood 117:3311-3319

Lin S, Staahl BT, Alla RK, Doudna JA (2014) Enhanced homologydirected human genome engineering by controlled timing of CRISPR/Cas9 delivery. eLife 3:e04766

Mali P, Yang L, Esvelt KM, Aach J, Guell M, DiCarlo JE, Norville JE, Church GM (2013) RNA-guided human genome engineering via Cas9. Science 339:823-826

Nanbo A, Sugden A, Sugden B (2007) The coupling of synthesis and partitioning of EBV's plasmid replicon is revealed in live cells. EMBO J 26:4252-4262

Okita K, Matsumura Y, Sato Y, Okada A, Morizane A, Okamoto S, Hong H, Nakagawa M, Tanabe K, Tezuka K et al (2011) A more efficient method to generate integration-free human iPS cells. Nat Methods 8:U409-U452

Yin H, Song CQ, Dorkin JR, Zhu LJ, Li Y, Wu Q, Park A, Yang J, Suresh S, Bizhanova A et al (2016) Therapeutic genome editing by combined viral and non-viral delivery of CRISPR system components in vivo. Nat Biotechnol 34:328-333

Yu JY, Hu KJ, Smuga-Otto K, Tian SL, Stewart R, Slukvin II, Thomson JA (2009) Human induced pluripotent stem cells free of vector and transgene sequences. Science 324:797-801 